\title{
Satisfacción laboral y estrés en teletrabajadores y trabajadores presenciales de una universidad estatal de Costa Rica
}

\author{
Lourdes Arce Espinoza1 ${ }^{1}$ y Karla Rojas Sáurez ${ }^{1}$ iD \\ 1. Universidad Estatal a Distancia, Vicerrectoría Ejecutiva, Servicio Médico UNED, 2050 Sabanilla, San José, Costa Rica; \\ larce@uned.ac.cr; krojas@uned.ac.cr
}

Recibido 7-VII-2020 • Corregido 21-IX-2020 • Aceptado 16-X-2020

DOI: https://doi.org/10.22458/urj.v12i2.3141

\begin{abstract}
Job satisfaction and stress in presential workers and tele-workers of a state university of Costa Rica". Introduction: Teleworking was introduced in the 1970 s as a result of the oil crisis and in response to the need for continuing economic activities. Decades later, advances in technology allowed the introduction of this modality globally. Many studies have shown that the benefits for organizations and employees are multiple; these include the increase in job satisfaction and a decrease in stress. Organizations have taken an active role in its implementation because satisfied and less stressed employees are more productive. Objective: Testing the hypotheses that, a) teleworkers are more satisfied, b) teleworkers perceive less stress. Methods: We surveyed 240 teleworkers and 224 presential workers. Results: Teleworkers have greater satisfaction and less stress. Conclusion: Teleworking is, in this institution, a satisfactory option to presential work regarding levels of satisfaction and stress.
\end{abstract}

Keywords: quality of life, well-being, health, labor flexibility, social commitment, telework, face-to-face work.
RESUMEN. Introducción: el teletrabajo se introdujo en la década de 1970 como resultado de la crisis del petróleo y en respuesta a la necesidad de continuar las actividades económicas. Décadas más tarde, los avances tecnológicos permitieron la introducción de esta modalidad a nivel mundial. Numerosos estudios han demostrado que los beneficios para las organizaciones y los empleados son múltiples; estos incluyen el aumento de la satisfacción laboral y una disminución del estrés. Las organizaciones han asumido un papel activo en su implementación porque los empleados satisfechos y menos estresados son más productivos. Objetivo: evaluar las hipótesis de que, a) los teletrabajadores están más satisfechos, b) los teletrabajadores perciben menos estrés. Métodos: Encuestamos a 240 teletrabajadores y 224 trabajadores presenciales. Resultados: Los teletrabajadores tienen mayor satisfacción y menos estrés. Conclusión: El teletrabajo es, en esta institución, una opción adecuada al trabajo presencial en cuanto a niveles de satisfacción y estrés.

Palabras clave: calidad de vida, bienestar, salud, flexibilidad laboral, compromiso social, teletrabajo, trabajo presencial.

El trabajo presencial ha sido durante mucho tiempo la única modalidad laboral en las organizaciones, la presencialidad en el centro de trabajo fue por años primordial para mantener la dinámica empleador-empleado. Con las crisis petroleras y los avances tecnológicos se vislumbró una innovadora forma de laborar a distancia, dando paso a lo que se conoce en la actualidad como teletrabajo (TT). Desde su incursión a nivel mundial, el TT ha ganado aceptación, cada día más organizaciones se suman a esta modalidad debido al beneficio que genera a la empresa en cuanto a la disminución de gastos de infraestructura, tecnología y servicios; su implementación en las organizaciones, ha sido de forma paulatina, pero con crecimiento ascendente (Sánchez, Montenegro, \& Medina, 2019; Brindusa, Cozzolino, \& Lacuesta, 2020).

El Teletrabajo como modalidad alternativa laboral en las empresas data de los años 70's; Jack Nilles propuso llevar el trabajo al empleado, bajo el término "telecommuting", sin embargo, para su implementación encontró dificultades tecnológicas propias de la época. Con los avances tecnológicos, el surgimiento de internet y una mayor disponibilidad de equipo electrónico, el TT podría dejar de ser la forma innovadora a nivel laboral para llegar a ser una modalidad ordinaria o habitual de trabajar (Pérez, 2010; Maresca, 2007; Moeckel, 2016). 
Aunque existen diversas definiciones acerca del término de TT, todas concuerdan en que es una modalidad donde media la distancia y las telecomunicaciones. En estas se incluirían: la informática, la telemática y las telecomunicaciones. Aunque existen variaciones del TT, cada organización determinará cuál o cuáles variaciones puede adoptar para el logro de sus objetivos y de acuerdo con las capacidades y habilidades de sus colaboradores (Saco, 2007; Caamaño, 2010; Vries \& Tummers, 2019).

De forma general se puede afirmar que el TT brinda al trabajador más beneficios que la modalidad presencial en cuanto a la calidad de vida, dentro de las ventajas que ofrece están: mejor organización del tiempo y las funciones, aumento de la productividad, mayor autonomía, conciliación del tiempo familiar, disminución en desplazamientos, inclusión de poblaciones con pocas oportunidades laborales, disminución de la huella de carbono, disminución del estrés y aumento en el nivel de satisfacción (Maresca, 2007; Martínez, Pérez, De Luis, \& Vela, 2009).

Estudios a nivel mundial relacionan una mejor calidad de vida en los trabajadores con alguna modalidad de $\mathrm{TT}$; las organizaciones se han preocupado en brindar al empleado otras remuneraciones que tengan que ver con la satisfacción y el manejo del estrés y no solo en el salario percibido (Durán, 2010). Estos estudios afirman que el nivel de estrés y la satisfacción en general, es mayor en los teletrabajadores que los trabajadores presenciales. Aunque la satisfacción es producto de diferentes factores que conforman los ejes de vida de los individuos, también es cierto que el ser humano es integral. La satisfacción laboral repercute en la satisfacción personal y viceversa, generando mayor satisfacción y disminución en el nivel de estrés (Martínez et al., 2009; López, Pérez-Simon, Nagham-Ngwessitcheu, Vázquez-Ubago, 2014).

En cuanto al estrés, es necesario acotar que, si bien es cierto, existen factores que generan estrés en los individuos, es cierto también que no todos los individuos reaccionan igual a estos estímulos. Sin embargo, de forma general, podría afirmarse que dentro de los factores laborales que generan estrés se encuentran, los desplazamientos y congestionamientos viales, la poca flexibilidad horaria y la pérdida de tiempo en desplazamientos. Dichos factores se encuentran ausentes en el TT, por lo que, al anular estos factores, el nivel de estrés percibido es menor (Maresca, 2007; Martínez et al., 2009; López et al., 2014).

Los estudios realizados acerca de la satisfacción laboral y el nivel de estrés datan de al menos un siglo; las organizaciones han invertido recursos en determinar factores o variables que aumenten la satisfacción laboral y disminuyan el nivel de estrés, esto puesto se ha podido concluir que un empleado satisfecho y sin estrés aumenta: la productividad, la creatividad, la toma de decisiones, además de su identificación con la compañía, por lo cual tendrá un mejor desempeño y esto tendrá impacto positivo económico en la empresa (Moscoso, 2014; Oviedo \& Vásquez, 2014; Núñez \& Quirós-Ramírez, 2016).

Este estudio ofrece información científica original sobre este tema en el ámbito que se realizó; los resultados pueden ser utilizados en otros estudios y en ambientes similares. El objetivo principal del estudio es comprobar las hipótesis alternativas de que, a) los teletrabajadores perciben mayor grado de satisfacción con respecto a la percepción de los trabajadores presenciales b) los teletrabajadores perciben un menor nivel de estrés con respecto a los trabajadores presenciales. 


\section{MATERIALES Y MÉTODOS}

Instrumento: El instrumento fue diseñado para aplicarlo en línea utilizándose el software LIMESURVEY. Consta de siete secciones: 1. Datos personales, 2. Datos de salud, 3. Nutrición, 4. Actividad física, 5. Calidad de vida, 6. Estrés, 7. Uso de equipo y seguridad. Sin embargo, para los efectos del presente estudio solo se consideran los resultados de las secciones: datos personales, calidad de vida y estrés. El instrumento fue validado por juicio de expertos, se envió a 30 personas escogidas al azar para poder determinar de manera independiente la relevancia y congruencia del contenido del cuestionario con el contenido teórico, la claridad en la redacción y el sesgo o tendenciosidad en la formulación de los ítems, es decir, si sugieren o no una respuesta (HernándezSampieri, Fernández, \& Baptista, 2014).

Procedimiento: Con respecto a los teletrabajadores, se solicitó al Programa de Teletrabajo la lista de los funcionarios teletrabajadores que tuvieran al menos un año de estar en esta modalidad de trabajo; posteriormente se envió un correo personalizado que incluía la invitación para participar en el estudio, en este correo se indicaba un enlace para acceder el cuestionario y responderlo a través de la web. Con respecto a los trabajadores presenciales, se solicitó a la Oficina de Recursos Humanos, la lista de funcionarios activos, con cuenta de correo electrónico y que al menos tuvieran un año de laborar en la institución. Se eliminaron del listado los Teletrabajadores y posteriormente se le asignó un número a cada funcionario de la lista y con un programa en línea generador de números al azar, se determinó un listado de 350 funcionarios de funcionarios presenciales, a los cuales se les envió un correo personalizado que incluía la invitación para participar en el estudio. Durante el periodo en que se mantuvo activo el cuestionario se enviaron tres recordatorios a los funcionarios que no lo habían contestado con el fin de motivar la participación. Se envió a 300 funcionarios teletrabajadores y 350 funcionarios presenciales, lográndose obtener un $80 \%$ de respuesta en la población teletrabajadora y $64 \%$ del grupo de trabajadores presenciales; se mantuvo activo desde el 01 de octubre de 2019 al 14 de diciembre del 2019.

Análisis estadístico: Los datos fueron tabulados inicialmente en un archivo tipo hoja de cálculo y luego se elaboró una base de datos utilizándose el software IBM SPSS Statisctics versión 20, mediante el cual se realizó el análisis descriptivo que comprende la elaboración distribuciones de frecuencia, tablas de contingencia y cálculo de valores promedio. Para realizar los contrastes de hipótesis se aplicaron pruebas no paramétricas; en el caso de variables ordinales se utilizó el estadístico $U$ de Mann-Whitney y para variables nominales el coeficiente de contingencia Chicuadrado.

Con el propósito de verificar la fiabilidad de los constructos diseñados para medir la percepción de la satisfacción laboral y el estrés, se realiza un análisis de fiabilidad por medio del estadístico alfa de Cronbach (ver apéndices 1-2).

\section{RESULTADOS}

En relación con las características sociodemográficas, laborales de los funcionarios teletrabajadores se obtuvo que, existe mayor presencia del sexo femenino, la mayoría de los funcionarios tienen entre 31 a 40 años y más, son casados, no tienen hijos. En mayor proporción se cuenta con formación universitaria completa y cuentan con más de 11 años de antigüedad laboral. En su mayoría ocupan puestos profesionales y laboran en la Vicerrectoría Académica.

En relación con las características sociodemográficas, laborales de los funcionarios presenciales, se obtuvo que, existe mayor presencia del sexo femenino, la mayoría de los 
funcionarios tienen menos de 30 años, son divorciados, separados o viudos, tienen dos o más hijos. En mayor proporción se cuenta con formación universitaria completa y cuentan con menos de 10 años de antigüedad laboral. En su mayoría ocupan puestos administrativos y laboran en la Vicerrectoría Académica.

Los resultados apoyan la hipótesis alternativa de que los funcionarios teletrabajadores perciben mayor satisfacción, al verificarse una diferencia significativa con relación a la modalidad de trabajo ( $U$ de Mann-Whitney $(\mathrm{n} 1=241, \mathrm{n} 2=223)=22251,5, p=0,001)$ (figura 1); y con respecto a la hipótesis de que los funcionarios teletrabajadores perciben menos estrés que los trabajadores presenciales, también se comprueba una diferencia significativa entre las modalidades ( $U$ de MannWhitney $(\mathrm{n} 1=241, \mathrm{n} 2=223)=20864,5, p=0,000$ (figura 2). Se constata que ambos índices construidos poseen alta fiabilidad (alfa de Cronbach $=0,899$ ) (Ver apéndices 1-2). Por tanto, puede considerarse que el teletrabajo tiene un efecto positivo en ambos aspectos.

\section{DISCUSIÓN}

La incorporación creciente de la mujer en el ámbito laboral, ha generado cambios a nivel estructural en las organizaciones y los hogares; la búsqueda de la conciliación del crecimiento profesional y laboral con la vida familiar, ha permitido una serie de ajustes que permitan dicha conciliación; la opción de TT es una estrategia adoptada por las organizaciones, cuyo efecto rebote ha generado armonía de la vida laboral y la vida familiar y cuya opción de trabajo se hace más atractiva para las mujeres. En diversos estudios europeos se ha determinado que las mujeres optan por la modalidad de TT más que los hombres, lo cual se explica basándose en que las mujeres generalmente buscan en el TT la conciliación del trabajo y el hogar, así como mayor disponibilidad del tiempo para la vida familiar, mientras que en los hombres este factor parece no ser determinante (Caamaño, 2010; Tapasco \& Giraldo, 2016; Secretaría de Salud Laboral y Secretaría de las Mujeres, 2017; Vicente-Herrero, Torres, Torres, Ramírez, \& Capdevila, 2018).

Generalmente, a mayor edad y experiencia laboral, hay mayor probabilidad de optar por el TT. Los estudios demuestran que los trabajadores mayores de 30 años, optan por esta modalidad en comparación con las poblaciones más jóvenes y esto está determinado por el perfil del trabajador el cual debe tener ciertas características, entre las que destacan: ser responsable, autónomo, proactivo, tener madurez laboral y habilidades de manejo de la tecnología, la mayoría de estas características se pueden apreciar a través del tiempo de servicio y por medio del desempeño del trabajador; es por esto que, a mayor edad y antigüedad en el puesto, existen mayores posibilidades que se apruebe esta modalidad en los empleados (Myers, 2005; Vayre, 2019; Vries \& Tummers, 2019; Brindusa et al., 2020). Otro factor importante es que, a mayor edad, las personas buscan mejorar la calidad de vida a través de la satisfacción y disminución del estrés, el TT ha demostrado que contribuye en ambos de forma significativa, al establecer un equilibrio en el tiempo de desarrollo personal, familiar y social (Pinquart \& Sörensen; 2001; Brindusa et al., 2020; Lampert \& Poblete, 2018).

La preparación académica también influye, la mayoría de los puestos profesionales, tienen mayor opción de realizar $\Pi$ T. Diversos estudios indican que la preparación académica está ligada a la opción de TT, debido a que los puestos profesionales, generalmente, poseen funciones administrativas, ejecutivas y organizativas, donde no es necesaria la presencialidad, ya que estas funciones están más ligadas a la tecnología, mientras que, para los puestos administrativos, técnicos y otros, podría ser menos accesible optar por esta modalidad, por la naturaleza propia de sus funciones (Urzúa \& Caqueo-Urízar, 2012; Vries \& Tummers, 2019). Asimismo, las organizaciones indican que la mayoría de los empleados en esta modalidad, son aquellos que cumplen como "funcionario calificado", es decir, es un funcionario de alto conocimiento, gran experiencia y 
preparación académica, lo que permite teletrabajadores altamente preparados y con las habilidades necesarias para lograr los objetivos y proyectos laborales (Vayre, 2019;OIT, 2016; CIDTT, 2017).

\section{CUADRO 1}

Distribución porcentual de las características sociodemográficas y laborales de los funcionarios según modalidad de trabajo

\begin{tabular}{|c|c|c|}
\hline Característica & $\begin{array}{c}\text { Teletrabajador } \\
(\%)\end{array}$ & $\begin{array}{c}\text { Trabajador } \\
\text { presencial (\%) }\end{array}$ \\
\hline \multicolumn{3}{|l|}{ Sexo } \\
\hline Femenino & 75,1 & 53,4 \\
\hline Masculino & 24,9 & 46,6 \\
\hline \multicolumn{3}{|l|}{ Grupo de edad } \\
\hline Menos de 30 & 4,6 & 53,4 \\
\hline 31 a 40 & 42,7 & 46,6 \\
\hline 41 a 50 & 31,5 & 0,0 \\
\hline Más de 50 & 21,2 & 0,0 \\
\hline \multicolumn{3}{|l|}{ Estado civil } \\
\hline Soltero & 27,4 & 0,0 \\
\hline Casado & 48,1 & 11,7 \\
\hline Divorciado, separado, viudo & 14,5 & 59,6 \\
\hline Unión Libre & 10 & 28,7 \\
\hline \multicolumn{3}{|l|}{$\mathbf{N}^{\circ}$ de hijos(as) } \\
\hline Ninguno & 45,6 & 3,1 \\
\hline 1 hijo(a) & 24,9 & 32,7 \\
\hline 2 hijos(as) & 23,2 & 39,5 \\
\hline 3 o más hijos(as) & 6,2 & 19,2 \\
\hline \multicolumn{3}{|l|}{ Escolaridad } \\
\hline Universitaria completa & 95 & 62,8 \\
\hline Universitaria incompleta & 3,7 & 22.4 \\
\hline Otro & 1,3 & 14,8 \\
\hline \multicolumn{3}{|l|}{ Antigüedad laboral (años) } \\
\hline Menos de 10 & 41,5 & 50,2 \\
\hline 11 a 20 & 44,8 & 27,4 \\
\hline 21 a 30 & 10,0 & 13,9 \\
\hline Más de 30 & 3,7 & 8,5 \\
\hline \multicolumn{3}{|l|}{ Puesto } \\
\hline Personal profesional & 88,0 & 49,8 \\
\hline Personal administrativo & 12,0 & 50,2 \\
\hline \multicolumn{3}{|l|}{ Departamento } \\
\hline Rectoría & 12 & 12,6 \\
\hline Vicerrectoría Académica & 59,8 & 49,3 \\
\hline Vicerrectoría Ejecutiva & 14,5 & 30 \\
\hline Vicerrectoría Planificación & 9,1 & 3,1 \\
\hline Vicerrectoría Investigación & 4,6 & 5 \\
\hline
\end{tabular}


Satisfacción con el tipo de trabajo

Satisfacción con el sueldo

Posibilidad de ascenso

Reconocimiento de mi esfuerzo

Apoyo de mis jefes

Apoyo de mis compañeros

Posibilidad de ser proactivo

Devolución de resultados de mi trabajo

Posibilidad de expresar mi opinión

Compromiso de mi jefatura por mejorar la calidad de vida profesional

Se cuenta con autonomía y libertad de decisión

Variedad de funciones en mi carga de trabajo

Mi opinión es tomado en cuenta TT

Índice de satisfacción laboral global

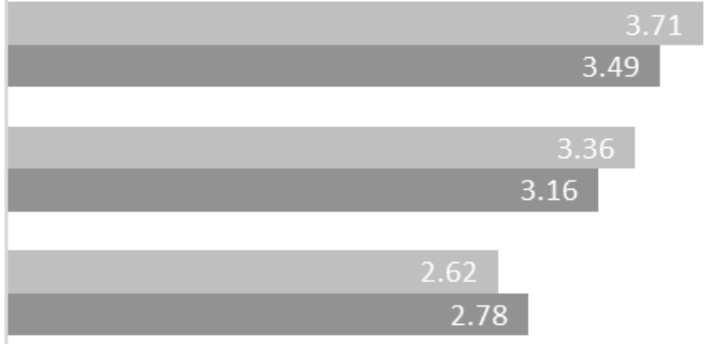

2.78

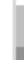

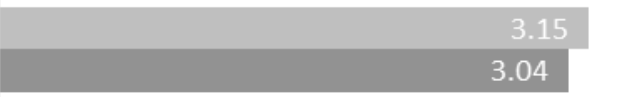

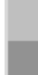

3.04
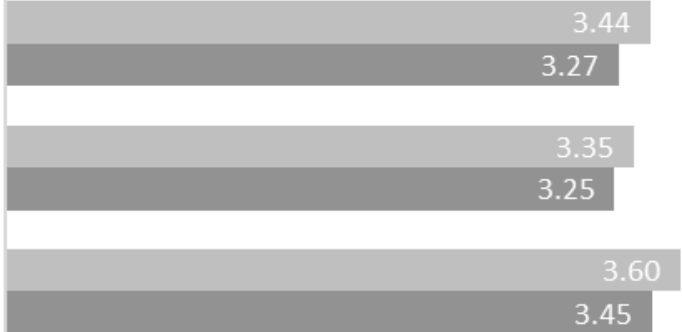

3.45
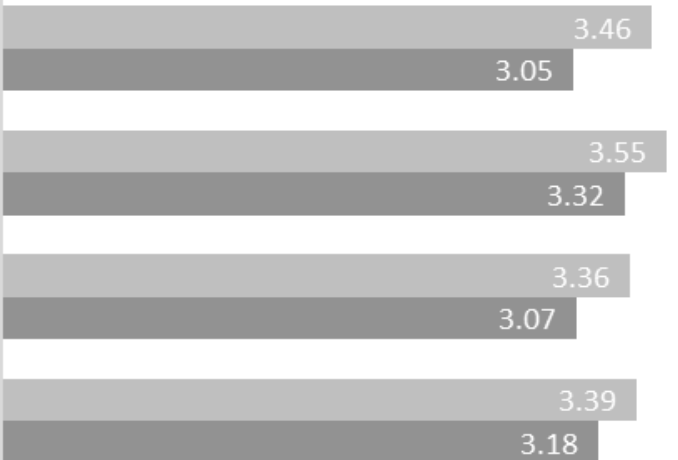

3.18
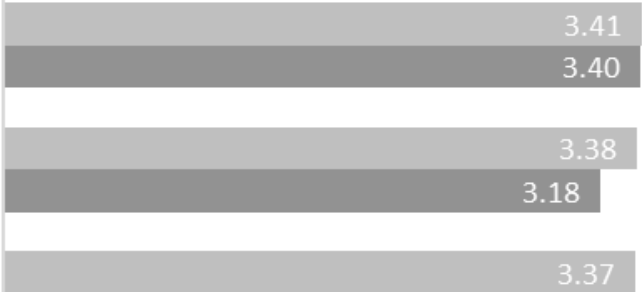

\section{Teletrabajador $\square$ Presencial}

Fig. 1. Percepción de la satisfacción según la modalidad de trabajo, valores promedio con base en escala de 1 a 4

Se comprobó a través de las hipótesis alternativas que la satisfacción es mayor y el nivel de estrés es menor en los teletrabajadores que en los trabajadores presenciales. Los beneficios del TT que suman para una mejor calidad de vida tienen que ver con: disminución del tiempo de traslados, menos pérdida de tiempo en congestionamientos viales, ahorro de dinero en traslados, una mejor conciliación de la vida familiar y personal, así como el aumento de la productividad, generando empleados más satisfechos y menos estresados (Moeckel, 2016; Núñez \& Quirós-Ramírez, 2016; 
Vries \& Tummers, 2019; Brindusa et al., 2020). La percepción de estos factores en términos de calidad de vida, pone al TT en ventaja con respecto a la modalidad presencial; y aunque algunos beneficios del TT corresponden también a aspectos de ahorro económico o productivos para el trabajador, influyen en la percepción de la mejora de calidad de vida, aumentando la satisfacción y disminuyendo el estrés (Uchida, Norasakkunkit, \& Kitayama 2004; Urzúa \& Caqueo-Urízar, 2012; Arce, 2015; Arce \& Sáurez, 2019; Vayre, 2019).

Si bien los factores que influyen en la satisfacción y el estrés tiene que ver también con las características personales y pueden variar de un día a otro por diferentes circunstancias, la posibilidad que ofrece el TT de contribuir en estas dos condicionantes de calidad de vida (satisfacción y estrés) son invaluables, en un mundo convulso, donde se pide la mayor productividad, se está expuesto a una gran cantidad de información, se pide altos conocimientos en múltiples campos; cada acción que disminuya el impacto de la vida agitada, constituye un aporte invaluable a la calidad de vida (Urzúa \& Caqueo-Urízar, 2012; Moeckel, 2016; Arce, 2015; Arce \& Sáurez, 2019; Vries \& Tummers, 2019). Se ha demostrado que el TT contribuye a la calidad de vida, disminuyendo el nivel de estrés y aumentando la satisfacción de los teletrabajadores en esta universidad estatal de Costa Rica, por lo cual se espera que el TT pase de ser algo innovador a ser la rutina en esta universidad estatal.

\section{AGRADECIMIENTOS}

A Julián Monge de la Vicerrectoría de Investigación UNED, por su apoyo, guía y acertados consejos en el desarrollo de esta investigación. A Ligia Bermúdez Mesén de la Vicerrectoría de Investigación UNED, por su apoyo en esta investigación. A todos los funcionarios que completaron el instrumento.

\section{ÉTICA, CONFLICTO DE INTERESES Y DECLARACIÓN DE FINANCIAMIENTO}

Los autores declaramos haber cumplido con todos los requisitos éticos y legales pertinentes, tanto durante el estudio como en el manuscrito; que no hay conflictos de interés de ningún tipo, no existen fuentes financieras que declarar. Asimismo, estamos de acuerdo con la versión editada final del documento. El respectivo documento legal firmado se encuentra en los archivos de la revista.

La contribución de los autores es como se detalla a continuación: L.A.E.: Elaboración del proyecto de investigación y participación activa durante todas las etapas de la Investigación: elaboración del proyecto e instrumento, sensibilización de la población, recolección de la información, tabulación, análisis estadístico y elaboración del artículo. K.R.S.: Supervisión y revisión del proyecto de Investigación en todas las etapas. 


\section{REFERENCIAS}

Arce, L. (2015). Nivel de satisfacción con su vida de los funcionarios de una universidad estatal de Costa Rica. Research Journal of the Costa Rican Distance Education University, 7(2), 131-135.

Arce, L., \& Sáurez, K. (2019). Satisfacción con la vida, el trabajo, y el país, en funcionarios de una universidad costarricense: comparación de sede central y centros regionales. UNED Research Journal, 11(3), 320- 326.

Brindusa, A., Cozzolino, M., \& Lacuesta, A. (2020). El teletrabajo en España. Boletín económico: Banco de España, 18(1), 118.

Caamaño, R. E. (2010). El teletrabajo como una alternativa para promover y facilitar la conciliación de responsabilidades laborales y familiares. Revista de Derecho de la Pontificia Universidad Católica de Valparaíso , 35(2), 79-105.

Centro Internacional para el desarrollo del Teletrabajo (CIDTT). (2017). Primer Informe sobre el estado del teletrabajo en Costa Rica. San José, Costa Rica: CIIDTT.

Durán, M. M. (2010). Bienestar psicológico, el estrés y la calidad de vida en el contexto laboral. Revista Nacional de Administración, 1(1), 71-84.

Hernández-Sampieri, R., Fernández Collado, C., \& Baptista Lucio, P. (2014).Metodología de la investigación. México D.F.: McGraw-Hill.

Lampert, G. M. P., \& Poblete, M. (2018). Efectos positivos y negativos del teletrabajo sobre la salud. Asesoría Técnica Parlamentaria, 1, 1-9.

López, N. W., Pérez-Simon, M. C., Nagham-Ngwessitcheu, E. G., \& Vázquez-Ubago, M. (2014). Teletrabajo, un enfoque desde la perspectiva de la salud laboral. Medicina y Seguridad del Trabajo, 60(236), 587- 599.

Maresca, G. (2007). Un paradigma diferente del management: Teletrabajo. Revista de Ciencias Empresariales, 2, 7-17.

Martínez, S. Á., Pérez, P. M., De Luis, C. P., \& Vela, J. J. (2009). Teletrabajo, flexibiidad de recursos humanos y resultados de la empresa. AIMS-Management, 11(2), 52-79.

Moeckel, R. (2016). Teleworking from home: Modeling the Impact of Telework on Transportation and Land Use. Science Diet, 26, 207-214.

Moscoso, M. (2014). El estudio científico del estrés crónico en neurociencias y psicooncología. Revista Persona, 17, 53-70.

Myers, D. G. (2005). Psicología. Madrid, España. Médica Panamericana.

Núñez, A. N., \& Quirós-Ramírez, R. A. (2016). El teletrabajo en Costa Rica, un estudio exploratorio sobre su implementación en el Gran Área Metropolitana. Hacia la Sociedad de la Información y el Conocimiento en Costa Rica: Informe 2017. (295-332). San José, Costa Rica: PROSIC-UCR.

Organización Internacional de Trabajo (OIT). (2016). Las dificultades y oportunidades del teletrabajo para los trabajadores y empleadores en los sectores de servicios de tecnología de la información y las comunicaciones (TIC) y financieros. Ginebra: Departamento de Políticas Sectoriales.

Oviedo, V. A., \& Vásquez, F. N. (2014). El teletrabajo: una estrategia de motivación. Revista Nacional de Administración, $5(2), 41-56$.

Pérez, S. C. (2010). El teletrabajo: ¿Más libertad o una nueva forma de esclavitud para los trabajadores? . Revista de Internet, Derecho y Política, 11, 22-33. 
Pinquart, M., \& Sörensen,S. (2001). Gender differences in self-concept and psychological well being in old age: A MetaAnalysis. Journal of Gerontology, Series B, 56(4), 195-216.

Saco, B. R. (2007). El teletrabajo. Derecho PUCP, 60, 325-350.

Sánchez, P. G., Montenegro, R. A., \& Medina, C. P. (2019). Teletrabajo una propuesta de innovación en productividad empresarial. Digital Publisher, 5(1), 91-104.

Secretaría de Salud Laboral y Secretaría de las Mujeres FSC-CCOO. (2017). El teletrabajo desde la perspectiva de género y salud Ocupacional. Recuperado de https://www.pv.ccoo.es/5b27ca809dcc63aaf471267a8677a78e000050.pdf

Tapasco, O., \& Giraldo, J. (2016). Factores Asociados a la Disposición por el Teletrabajo entre Docentes Universitarios. Ciencia y trabajo, 18(56), 87-93.

Uchida, Y., Norasakkunkit. V., \& Kitayama, S. (2004). Cultural constructions of happiness: Theory and empirical evidence. Journal of Happiness Studies, 5, 223-229.

Urzúa, M.A., \& Caqueo-Urízar. A. (2012). Calidad de vida: una revisión teórica del concepto. Revista Terapia Psicológica, 30(1), 61-71.

Vayre, É. (2019). Impacts of telework on the worker and his professional, family and social spheres. Le travail humain, 82(1), 1-39

Vicente-Herrero, T., Torres, A. I., Torres, V. A., Ramírez, I. d., \& Capdevila, G. L. (2018). El Teletrabajo en salud laboral. Revista CES Derecho, 9(2), 287-297.

Vries, H., \& Tummers, L. (2019). The Benefits of Teleworking in the Public Sector: Reality or Rhetoric? Review of Public Personnel Administration, 39(4), 570-593. 
APÉNDICE DIGITAL

APÉNDICE 1

Resultados de Alfa Cronbach para el índice nivel de satisfacción

Correlación Alfa de

elemento- Cronbach si

total se elimina

\begin{tabular}{|c|c|c|c|c|}
\hline Item & Media & $\mathbf{N}$ & corregida & el elemento \\
\hline $\begin{array}{l}\text { Satisfacción con el tipo } \\
\text { de trabajo }\end{array}$ & 3,61 & 464 & ,543 & 890 \\
\hline $\begin{array}{l}\text { Satisfacción con el } \\
\text { sueldo }\end{array}$ & 3,26 & 464 & ,389 & ,896 \\
\hline Posibilidad de ascenso & 2,70 & 464 & 370 & 900 \\
\hline $\begin{array}{l}\text { Reconocimiento de mi } \\
\text { esfuerzo }\end{array}$ & 3,09 & 464 & ,670 & 884, \\
\hline Apoyo de mis jefes & 3,36 & 464 & ,728 & ,881 \\
\hline $\begin{array}{l}\text { Apoyo de mis } \\
\text { compañeros }\end{array}$ & 3,30 & 464 & ,517 & ,891 \\
\hline $\begin{array}{l}\text { Posibilidad de ser } \\
\text { proactivo }\end{array}$ & 3,53 & 464 & 623 & ,887 \\
\hline $\begin{array}{l}\text { Devolución de resultados } \\
\text { de mi trabajo }\end{array}$ & 3,27 & 464 & ,572 & ,889 \\
\hline $\begin{array}{l}\text { Posibilidad de expresar } \\
\text { mi opinión }\end{array}$ & 3,44 & 464 & ,720 & 882 \\
\hline $\begin{array}{l}\text { Compromiso de mi } \\
\text { jefatura por mejorar la } \\
\text { calidad de vida } \\
\text { profesional }\end{array}$ & 3,22 & 464 & 697 & 882 \\
\hline $\begin{array}{l}\text { Se cuenta con autonomía } \\
\text { y libertad de decisión }\end{array}$ & 3,29 & 464 & ,721 & ,881 \\
\hline $\begin{array}{l}\text { Variedad de funciones en } \\
\text { mi carga de trabajo }\end{array}$ & 3,40 & 464 & ,527 & 0 \\
\hline $\begin{array}{l}\text { Mi opinión es tomado en } \\
\text { cuenta TT }\end{array}$ & 3,29 & 464 & ,749 & , OC \\
\hline
\end{tabular}




\section{APÉNDICE 2}

Resultados de Alfa Cronbach para el índice de nivel de estrés

\begin{tabular}{|c|c|c|c|c|}
\hline Item & Media & $\mathbf{N}$ & $\begin{array}{l}\text { Correlación } \\
\text { elemento- } \\
\text { total } \\
\text { corregida }\end{array}$ & $\begin{array}{c}\text { Alfa de } \\
\text { Cronbach si } \\
\text { se elimina el } \\
\text { elemento }\end{array}$ \\
\hline $\begin{array}{l}\text { Imposibilidad de conciliar el } \\
\text { sueño }\end{array}$ & 3,13 & 464 & 623 & 900 \\
\hline Dolor de cabeza frecuente & 2,90 & 464 & ,668 & ,898 \\
\hline $\begin{array}{l}\text { Problemas } \\
\text { gastrointestinales }\end{array}$ & 3,03 & 464 & 587, & 902 \\
\hline $\begin{array}{l}\text { Sensación de cansancio } \\
\text { extremo }\end{array}$ & 3,67 & 464 & 732 & 895 \\
\hline $\begin{array}{l}\text { Tendencia a comer, beber, } \\
\text { fumar o tomar más de lo } \\
\text { habitual }\end{array}$ & 2,37 & 464 & ,546 & 904 \\
\hline $\begin{array}{l}\text { Disminución de interés } \\
\text { sexual }\end{array}$ & 2,69 & 464 & 519, & 905 \\
\hline $\begin{array}{l}\text { Respiración entrecortada o } \\
\text { sensación de ahogo }\end{array}$ & 2,14 & 464 & ,670 & 898 \\
\hline Disminución del apetito & 2,04 & 464 &, 559 & ,903 \\
\hline Temblores musculares & 2,25 & 464 & ,661 & ,898 \\
\hline $\begin{array}{l}\text { Sensación de dolor en } \\
\text { diferentes partes del } \\
\text { cuerpo }\end{array}$ & 2,72 & 464 & 703, & 896 \\
\hline $\begin{array}{l}\text { Pocas ganas de levantarse } \\
\text { en las mañanas }\end{array}$ & 2,90 & 464 & 723 & 895 \\
\hline $\begin{array}{l}\text { Tendencia a sudoración y } \\
\text { palpitaciones }\end{array}$ & 2,09 & 464 & ,648 & ,899 \\
\hline
\end{tabular}

